\title{
Search for flavor-changing neutral current and lepton-flavor violating decays of $\mathbf{D}^{0} \rightarrow \ell^{+} \ell^{-}$
}

B. Aubert,${ }^{1}$ R. Barate,${ }^{1}$ D. Boutigny,${ }^{1}$ F. Couderc,${ }^{1}$ J.-M. Gaillard, ${ }^{1}$ A. Hicheur, ${ }^{1}$ Y. Karyotakis,${ }^{1}$ J. P. Lees,${ }^{1}$ V. Tisserand, ${ }^{1}$ A. Zghiche, ${ }^{1}$ A. Palano, ${ }^{2}$ A. Pompili, ${ }^{2}$ J. C. Chen,${ }^{3}$ N. D. Qi,${ }^{3}$ G. Rong, ${ }^{3}$ P. Wang, ${ }^{3}$ Y. S. Zhu,${ }^{3}$ G. Eigen, ${ }^{4}$ I. Ofte ${ }^{4}$ B. Stugu, ${ }^{4}$ G. S. Abrams, ${ }^{5}$ A. W. Borgland,${ }^{5}$ A. B. Breon, ${ }^{5}$ D. N. Brown, ${ }^{5}$ J. Button-Shafer,${ }^{5}$ R. N. Cahn, ${ }^{5}$ E. Charles ${ }^{5}$ C. T. Day, ${ }^{5}$ M. S. Gill,${ }^{5}$ A. V. Gritsan, ${ }^{5}$ Y. Groysman,${ }^{5}$ R. G. Jacobsen, ${ }^{5}$ R. W. Kadel, ${ }^{5}$ J. Kadyk,${ }^{5}$ L. T. Kerth, ${ }^{5}$ Yu. G. Kolomensky, ${ }^{5}$ G. Kukartsev, ${ }^{5}$ G. Lynch, ${ }^{5}$ L. M. Mir, ${ }^{5}$ P. J. Oddone,${ }^{5}$

T. J. Orimoto, ${ }^{5}$ M. Pripstein,${ }^{5}$ N. A. Roe,${ }^{5}$ M. T. Ronan,${ }^{5}$ V. G. Shelkov,${ }^{5}$ W. A. Wenzel,${ }^{5}$ M. Barrett, ${ }^{6}$ K. E. Ford ${ }^{6}$ T. J. Harrison, ${ }^{6}$ A. J. Hart,${ }^{6}$ C. M. Hawkes ${ }^{6}$ S. E. Morgan,${ }^{6}$ A. T. Watson, ${ }^{6}$ M. Fritsch,${ }^{7}$ K. Goetzen,${ }^{7}$ T. Held,${ }^{7}$ H. Koch,${ }^{7}$ B. Lewandowski,${ }^{7}$ M. Pelizaeus, ${ }^{7}$ M. Steinke, ${ }^{7}$ J. T. Boyd ${ }^{8}$ N. Chevalier,${ }^{8}$ W. N. Cottingham, ${ }^{8}$ M. P. Kelly ${ }^{8}$ T. E. Latham,${ }^{8}$ F. F. Wilson,${ }^{8}$ T. Cuhadar-Donszelmann,${ }^{9}$ C. Hearty,${ }^{9}$ N. S. Knecht,${ }^{9}$ T. S. Mattison, ${ }^{9}$ J. A. McKenna, ${ }^{9}$ D. Thiessen,${ }^{9}$ A. Khan,${ }^{10}$ P. Kyberd,${ }^{10}$ L. Teodorescu, ${ }^{10}$ A. E. Blinov,${ }^{11}$ V. E. Blinov,${ }^{11}$

V. P. Druzhinin, ${ }^{11}$ V. B. Golubev, ${ }^{11}$ V. N. Ivanchenko, ${ }^{11}$ E. A. Kravchenko, ${ }^{11}$ A. P. Onuchin,${ }^{11}$ S. I. Serednyakov,${ }^{11}$ Yu. I. Skovpen, ${ }^{11}$ E. P. Solodov ${ }^{11}$ A. N. Yushkov ${ }^{11}$ D. Best,${ }^{12}$ M. Bruinsma, ${ }^{12}$ M. Chao,${ }^{12}$ I. Eschrich,${ }^{12}$ D. Kirkby, ${ }^{12}$ A. J. Lankford, ${ }^{12}$ M. Mandelkern,${ }^{12}$ R. K. Mommsen, ${ }^{12}$ W. Roethel,${ }^{12}$ D. P. Stoker,${ }^{12}$ C. Buchanan,${ }^{13}$

B. L. Hartfiel, ${ }^{13}$ S. D. Foulkes,${ }^{14}$ J. W. Gary ${ }^{14}$ B. C. Shen,${ }^{14}$ K. Wang,${ }^{14}$ D. del Re,${ }^{15}$ H. K. Hadavand, ${ }^{15}$ E. J. Hill, ${ }^{15}$ D. B. MacFarlane, ${ }^{15}$ H. P. Paar, ${ }^{15}$ Sh. Rahatlou, ${ }^{15}$ V. Sharma, ${ }^{15}$ J. W. Berryhill, ${ }^{16}$ C. Campagnari, ${ }^{16}$ B. Dahmes ${ }^{16}$ O. Long, ${ }^{16}$ A. Lu, ${ }^{16}$ M. A. Mazur, ${ }^{16}$ J. D. Richman, ${ }^{16}$ W. Verkerke,${ }^{16}$ T. W. Beck, ${ }^{17}$ A. M. Eisner, ${ }^{17}$

C. A. Heusch, ${ }^{17}$ J. Kroseberg, ${ }^{17}$ W. S. Lockman, ${ }^{17}$ G. Nesom, ${ }^{17}$ T. Schalk, ${ }^{17}$ B. A. Schumm, ${ }^{17}$ A. Seiden, ${ }^{17}$ P. Spradlin, ${ }^{17}$ D. C. Williams, ${ }^{17}$ M. G. Wilson, ${ }^{17}$ J. Albert, ${ }^{18}$ E. Chen, ${ }^{18}$ G. P. Dubois-Felsmann,,${ }^{18}$ A. Dvoretskii, ${ }^{18}$

D. G. Hitlin, ${ }^{18}$ I. Narsky, ${ }^{18}$ T. Piatenko, ${ }^{18}$ F. C. Porter, ${ }^{18}$ A. Ryd ${ }^{18}$ A. Samuel, ${ }^{18}$ S. Yang, ${ }^{18}$ S. Jayatilleke, ${ }^{19}$ G. Mancinelli, ${ }^{19}$ B. T. Meadows, ${ }^{19}$ M. D. Sokoloff, ${ }^{19}$ T. Abe,${ }^{20}$ F. Blanc, ${ }^{20}$ P. Bloom, ${ }^{20}$ S. Chen, ${ }^{20}$ W. T. Ford ${ }^{20}$ U. Nauenberg, ${ }^{20}$ A. Olivas,${ }^{20}$ P. Rankin, ${ }^{20}$ J. G. Smith,${ }^{20}$ J. Zhang, ${ }^{20}$ L. Zhang, ${ }^{20}$ A. Chen, ${ }^{21}$ J. L. Harton, ${ }^{21}$ A. Soffer, ${ }^{21}$ W. H. Toki, ${ }^{21}$ R. J. Wilson, ${ }^{21}$ Q. Zeng, ${ }^{21}$ D. Altenburg, ${ }^{22}$ T. Brandt,${ }^{22}$ J. Brose,${ }^{22}$ M. Dickopp,${ }^{22}$ E. Feltresi, ${ }^{22}$ A. Hauke, ${ }^{22}$ H. M. Lacker,${ }^{22}$ R. Müller-Pfefferkorn, ${ }^{22}$ R. Nogowski, ${ }^{22}$ S. Otto, ${ }^{22}$ A. Petzold, ${ }^{22}$ J. Schubert, ${ }^{22}$ K. R. Schubert,${ }^{22}$ R. Schwierz, ${ }^{22}$ B. Spaan, ${ }^{22}$ J. E. Sundermann, ${ }^{22}$ D. Bernard, ${ }^{23}$ G. R. Bonneaud, ${ }^{23}$

F. Brochard ${ }^{23}$ P. Grenier, ${ }^{23}$ S. Schrenk, ${ }^{23}$ Ch. Thiebaux, ${ }^{23}$ G. Vasileiadis, ${ }^{23}$ M. Verderi, ${ }^{23}$ D. J. Bard, ${ }^{24}$

P. J. Clark, ${ }^{24}$ D. Lavin,${ }^{24}$ F. Muheim,${ }^{24}$ S. Playfer,${ }^{24}$ Y. Xie,${ }^{24}$ M. Andreotti,${ }^{25}$ V. Azzolini,${ }^{25}$ D. Bettoni,${ }^{25}$ C. Bozzi ${ }^{25}$ R. Calabrese,${ }^{25}$ G. Cibinetto, ${ }^{25}$ E. Luppi,${ }^{25}$ M. Negrini, ${ }^{25}$ L. Piemontese,${ }^{25}$ A. Sarti, ${ }^{25}$ E. Treadwell,${ }^{26}$ F. Anulli, ${ }^{27}$ R. Baldini-Ferroli, ${ }^{27}$ A. Calcaterra, ${ }^{27}$ R. de Sangro, ${ }^{27}$ G. Finocchiaro, ${ }^{27}$ P. Patteri, ${ }^{27}$ I. M. Peruzzi, ${ }^{27}$ M. Piccolo, ${ }^{27}$ A. Zallo, ${ }^{27}$ A. Buzzo, ${ }^{28}$ R. Capra, ${ }^{28}$ R. Contri, ${ }^{28}$ G. Crosetti, ${ }^{28}$ M. Lo Vetere, ${ }^{28}$ M. Macri, ${ }^{28}$ M. R. Monge ${ }^{28}$ S. Passaggio, ${ }^{28}$ C. Patrignani, ${ }^{28}$ E. Robutti ${ }^{28}$ A. Santroni,${ }^{28}$ S. Tosi,${ }^{28}$ S. Bailey,${ }^{29}$

G. Brandenburg, ${ }^{29}$ K. S. Chaisanguanthum, ${ }^{29}$ M. Morii, ${ }^{29}$ E. Won, ${ }^{29}$ R. S. Dubitzky, ${ }^{30}$ U. Langenegger, ${ }^{30}$ W. Bhimji, ${ }^{31}$ D. A. Bowerman, ${ }^{31}$ P. D. Dauncey ${ }^{31}$ U. Egede, ${ }^{31}$ J. R. Gaillard, ${ }^{31}$ G. W. Morton, ${ }^{31}$ J. A. Nash, ${ }^{31}$ M. B. Nikolich, ${ }^{31}$ G. P. Taylor, ${ }^{31}$ M. J. Charles, ${ }^{32}$ G. J. Grenier, ${ }^{32}$ U. Mallik, ${ }^{32}$ J. Cochran, ${ }^{33}$ H. B. Crawley, ${ }^{33}$ J. Lamsa, ${ }^{33}$ W. T. Meyer, ${ }^{33}$ S. Prell, ${ }^{33}$ E. I. Rosenberg, ${ }^{33}$ A. E. Rubin, ${ }^{33}$ J. Yi,${ }^{33}$ M. Biasini, ${ }^{34}$ R. Covarelli, ${ }^{34}$ M. Pioppi, ${ }^{34}$ M. Davier, ${ }^{35}$ X. Giroux,${ }^{35}$ G. Grosdidier, ${ }^{35}$ A. Höcker, ${ }^{35}$ S. Laplace, ${ }^{35}$ F. Le Diberder, ${ }^{35}$ V. Lepeltier,${ }^{35}$ A. M. Lutz,${ }^{35}$ T. C. Petersen, ${ }^{35}$ S. Plaszczynski, ${ }^{35}$ M. H. Schune, ${ }^{35}$ L. Tantot,${ }^{35}$ G. Wormser, ${ }^{35}$ C. H. Cheng, ${ }^{36}$ D. J. Lange ${ }^{36}$ M. C. Simani, ${ }^{36}$ D. M. Wright,${ }^{36}$ A. J. Bevan, ${ }^{37}$ C. A. Chavez,${ }^{37}$ J. P. Coleman, ${ }^{37}$ I. J. Forster ${ }^{37}$ J. R. Fry,${ }^{37}$ E. Gabathuler, ${ }^{37}$ R. Gamet, ${ }^{37}$ D. E. Hutchcroft,,${ }^{37}$ R. J. Parry,${ }^{37}$ D. J. Payne, ${ }^{37}$ R. J. Sloane, ${ }^{37}$ C. Touramanis ${ }^{37}$ J. J. Back, ${ }^{38, *}$ C. M. Cormack, ${ }^{38}$ P. F. Harrison, ${ }^{38, *}$ F. Di Lodovico, ${ }^{38}$ G. B. Mohanty,${ }^{38, *}$ C. L. Brown, ${ }^{39}$ G. Cowan ${ }^{39}$ R. L. Flack, ${ }^{39}$ H. U. Flaecher, ${ }^{39}$ M. G. Green,${ }^{39}$ P. S. Jackson,${ }^{39}$ T. R. McMahon, ${ }^{39}$ S. Ricciardi,${ }^{39}$ F. Salvatore,${ }^{39}$ M. A. Winter ${ }^{39}$ D. Brown, ${ }^{40}$ C. L. Davis,${ }^{40}$ J. Allison, ${ }^{41}$ N. R. Barlow,${ }^{41}$ R. J. Barlow, ${ }^{41}$ P. A. Hart, ${ }^{41}$ M. C. Hodgkinson, ${ }^{41}$ G. D. Lafferty, ${ }^{41}$ A. J. Lyon, ${ }^{41}$ J. C. Williams, ${ }^{41}$ C. Chen, ${ }^{42}$

A. Farbin, ${ }^{42}$ W. D. Hulsbergen ${ }^{42}$ A. Jawahery ${ }^{42}$ D. Kovalskyi, ${ }^{42}$ C. K. Lae ${ }^{42}$ V. Lillard, ${ }^{42}$ D. A. Roberts ${ }^{42}$ G. Blaylock, ${ }^{43}$ C. Dallapiccola, ${ }^{43}$ K. T. Flood ${ }^{43}$ S. S. Hertzbach, ${ }^{43}$ R. Kofler, ${ }^{43}$ V. B. Koptchev, ${ }^{43}$ T. B. Moore, ${ }^{43}$ 
S. Saremi, ${ }^{43}$ H. Staengle, ${ }^{43}$ S. Willocq,${ }^{43}$ R. Cowan, ${ }^{44}$ G. Sciolla,${ }^{44}$ S. J. Sekula, ${ }^{44}$ F. Taylor, ${ }^{44}$ R. K. Yamamoto, ${ }^{44}$ D. J. J. Mangeol, ${ }^{45}$ P. M. Patel, ${ }^{45}$ S. H. Robertson, ${ }^{45}$ A. Lazzaro, ${ }^{46}$ V. Lombardo ${ }^{46}$ F. Palombo, ${ }^{46}$

J. M. Bauer, ${ }^{47}$ L. Cremaldi, ${ }^{47}$ V. Eschenburg, ${ }^{47}$ R. Godang, ${ }^{47}$ R. Kroeger, ${ }^{47}$ J. Reidy, ${ }^{47}$ D. A. Sanders, ${ }^{47}$

D. J. Summers ${ }^{47}$ H. W. Zhao, ${ }^{47}$ S. Brunet, ${ }^{48}$ D. Côté, ${ }^{48}$ P. Taras,${ }^{48}$ H. Nicholson,${ }^{49}$ N. Cavallo,${ }^{50,}{ }^{\dagger}$ F. Fabozzi,,${ }^{50,}{ }^{\dagger}$

C. Gatto,${ }^{50}$ L. Lista, ${ }^{50}$ D. Monorchio, ${ }^{50}$ P. Paolucci, ${ }^{50}$ D. Piccolo,${ }^{50}$ C. Sciacca,,${ }^{50}$ M. Baak, ${ }^{51}$ H. Bulten, ${ }^{51}$

G. Raven, ${ }^{51}$ H. L. Snoek, ${ }^{51}$ L. Wilden, ${ }^{51}$ C. P. Jessop, ${ }^{52}$ J. M. LoSecco,,${ }^{52}$ T. Allmendinger, ${ }^{53}$ K. K. Gan, ${ }^{53}$

K. Honscheid ${ }^{53}$ D. Hufnagel,${ }^{53}$ H. Kagan, ${ }^{53}$ R. Kass,${ }^{53}$ T. Pulliam, ${ }^{53}$ A. M. Rahimi,${ }^{53}$ R. Ter-Antonyan, ${ }^{53}$

Q. K. Wong, ${ }^{53}$ J. Brau,${ }^{54}$ R. Frey,${ }^{54}$ O. Igonkina, ${ }^{54}$ C. T. Potter,${ }^{54}$ N. B. Sinev,${ }^{54}$ D. Strom,${ }^{54}$ E. Torrence,${ }^{54}$

F. Colecchia, ${ }^{55}$ A. Dorigo ${ }^{55}$ F. Galeazzi, ${ }^{55}$ M. Margoni, ${ }^{55}$ M. Morandin, ${ }^{55}$ M. Posocco,${ }^{55}$ M. Rotondo, ${ }^{55}$

F. Simonetto, ${ }^{55}$ R. Stroili, ${ }^{55}$ G. Tiozzo,${ }^{55}$ C. Voci,${ }^{55}$ M. Benayoun, ${ }^{56}$ H. Briand ${ }^{56}$ J. Chauveau, ${ }^{56}$ P. David, ${ }^{56}$ Ch. de la Vaissière ${ }^{56}$ L. Del Buono ${ }^{56}$ O. Hamon, ${ }^{56}$ M. J. J. John, ${ }^{56}$ Ph. Leruste,${ }^{56}$ J. Malcles ${ }^{56}$ J. Ocariz ${ }^{56}$ M. Pivk, ${ }^{56}$ L. Roos, ${ }^{56}$ S. T'Jampens, ${ }^{56}$ G. Therin, ${ }^{56}$ P. F. Manfredi, ${ }^{57}$ V. Re,${ }^{57}$ P. K. Behera,${ }^{58}$ L. Gladney, ${ }^{58}$ Q. H. Guo, ${ }^{58}$ J. Panetta, ${ }^{58}$ C. Angelini, ${ }^{59}$ G. Batignani, ${ }^{59}$ S. Bettarini, ${ }^{59}$ M. Bondioli, ${ }^{59}$ F. Bucci, ${ }^{59}$ G. Calderini, ${ }^{59}$ M. Carpinelli, ${ }^{59}$ F. Forti, ${ }^{59}$ M. A. Giorgi,${ }^{59}$ A. Lusiani, ${ }^{59}$ G. Marchiori,${ }^{59}$ F. Martinez-Vidal,${ }^{59,} \ddagger$ M. Morganti, ${ }^{59}$ N. Neri ${ }^{59}$ E. Paoloni ${ }^{59}$ M. Rama,${ }^{59}$ G. Rizzo, ${ }^{59}$ F. Sandrelli, ${ }^{59}$ J. Walsh,${ }^{59}$ M. Haire ${ }^{60}$ D. Judd ${ }^{60}$ K. Paick ${ }^{60}$ D. E. Wagoner ${ }^{60}$ N. Danielson, ${ }^{61}$ P. Elmer, ${ }^{61}$ Y. P. Lau,${ }^{61}$ C. Lu, ${ }^{61}$ V. Miftakov, ${ }^{61}$ J. Olsen, ${ }^{61}$ A. J. S. Smith, ${ }^{61}$ A. V. Telnov,${ }^{61}$ F. Bellini, ${ }^{62}$ G. Cavoto, ${ }^{61,}{ }^{62}$ R. Faccini, ${ }^{62}$ F. Ferrarotto, ${ }^{62}$ F. Ferroni, ${ }^{62}$ M. Gaspero, ${ }^{62}$ L. Li Gioi,${ }^{62}$ M. A. Mazzoni,${ }^{62}$ S. Morganti, ${ }^{62}$ M. Pierini,${ }^{62}$ G. Piredda,${ }^{62}$ F. Safai Tehrani,${ }^{62}$ C. Voena, ${ }^{62}$ S. Christ,${ }^{63}$ G. Wagner, ${ }^{63}$ R. Waldi, ${ }^{63}$ T. Adye,${ }^{64}$ N. De Groot,${ }^{64}$ B. Franek,${ }^{64}$ N. I. Geddes,${ }^{64}$ G. P. Gopal,${ }^{64}$ E. O. Olaiya, ${ }^{64}$ R. Aleksan,${ }^{65}$ S. Emery, ${ }^{65}$ A. Gaidot,${ }^{65}$ S. F. Ganzhur, ${ }^{65}$ P.-F. Giraud, ${ }^{65}$ G. Hamel de Monchenault, ${ }^{65}$ W. Kozanecki, ${ }^{65}$ M. Legendre, ${ }^{65}$ G. W. London, ${ }^{65}$ B. Mayer, ${ }^{65}$ G. Schott, ${ }^{65}$ G. Vasseur, ${ }^{65}$ Ch. Yèche ${ }^{65}$ M. Zito, ${ }^{65}$ M. V. Purohit,${ }^{66}$ A. W. Weidemann, ${ }^{66}$ J. R. Wilson, ${ }^{66}$ F. X. Yumiceva, ${ }^{66}$ D. Aston, ${ }^{67}$ R. Bartoldus, ${ }^{67}$ N. Berger, ${ }^{67}$ A. M. Boyarski ${ }^{67}$ O. L. Buchmueller, ${ }^{67}$ R. Claus,${ }^{67}$ M. R. Convery ${ }^{67}$ M. Cristinziani, ${ }^{67}$ G. De Nardo, ${ }^{67}$ D. Dong, ${ }^{67}$ J. Dorfan, ${ }^{67}$ D. Dujmic, ${ }^{67}$ W. Dunwoodie,${ }^{67}$ E. E. Elsen, ${ }^{67}$ S. Fan, ${ }^{67}$ R. C. Field, ${ }^{67}$ T. Glanzman, ${ }^{67}$ S. J. Gowdy, ${ }^{67}$ T. Hadig, ${ }^{67}$ V. Halyo,${ }^{67}$ C. Hast, ${ }^{67}$ T. Hryn'ova, ${ }^{67}$ W. R. Innes, ${ }^{67}$ M. H. Kelsey, ${ }^{67}$ P. Kim, ${ }^{67}$ M. L. Kocian, ${ }^{67}$ D. W. G. S. Leith, ${ }^{67}$ J. Libby,${ }^{67}$ S. Luitz ${ }^{67}$ V. Luth, ${ }^{67}$ H. L. Lynch, ${ }^{67}$ H. Marsiske, ${ }^{67}$ R. Messner, ${ }^{67}$ D. R. Muller, ${ }^{67}$ C. P. O'Grady,${ }^{67}$ V. E. Ozcan, ${ }^{67}$ A. Perazzo, ${ }^{67}$ M. Perl, ${ }^{67}$ S. Petrak, ${ }^{67}$ B. N. Ratcliff, ${ }^{67}$ A. Roodman, ${ }^{67}$ A. A. Salnikov, ${ }^{67}$ R. H. Schindler, ${ }^{67}$ J. Schwiening, ${ }^{67}$ G. Simi, ${ }^{67}$ A. Snyder ${ }^{67}$ A. Soha ${ }^{67}$ J. Stelzer ${ }^{67}$ D. Su,${ }^{67}$ M. K. Sullivan, ${ }^{67}$ J. Va'vra ${ }^{67}$ S. R. Wagner, ${ }^{67}$ M. Weaver ${ }^{67}$ A. J. R. Weinstein, ${ }^{67}$ W. J. Wisniewski, ${ }^{67}$ M. Wittgen,${ }^{67}$ D. H. Wright,${ }^{67}$ A. K. Yarritu, ${ }^{67}$ C. C. Young, ${ }^{67}$ P. R. Burchat, ${ }^{68}$ A. J. Edwards, ${ }^{68}$ T. I. Meyer, ${ }^{68}$ B. A. Petersen, ${ }^{68}$ C. Roat,${ }^{68}$ S. Ahmed, ${ }^{69}$ M. S. Alam, ${ }^{69}$ J. A. Ernst, ${ }^{69}$ M. A. Saeed, ${ }^{69}$ M. Saleem, ${ }^{69}$ F. R. Wappler, ${ }^{69}$ W. Bugg, ${ }^{70}$ M. Krishnamurthy, ${ }^{70}$ S. M. Spanier, ${ }^{70}$ R. Eckmann, ${ }^{71}$ H. Kim,${ }^{71}$ J. L. Ritchie,${ }^{71}$ A. Satpathy,${ }^{71}$ R. F. Schwitters,${ }^{71}$ J. M. Izen, ${ }^{72}$ I. Kitayama, ${ }^{72}$ X. C. Lou,${ }^{72}$ S. Ye ${ }^{72}$ F. Bianchi, ${ }^{73}$ M. Bona,${ }^{73}$ F. Gallo, ${ }^{73}$ D. Gamba,${ }^{73}$ L. Bosisio, ${ }^{74}$ C. Cartaro, ${ }^{74}$ F. Cossutti,${ }^{74}$ G. Della Ricca, ${ }^{74}$ S. Dittongo,${ }^{74}$ S. Grancagnolo, ${ }^{74}$ L. Lanceri, ${ }^{74}$ P. Poropat,${ }^{74,} \S$ L. Vitale,${ }^{74}$ G. Vuagnin, ${ }^{74}$ R. S. Panvini, ${ }^{75}$ Sw. Banerjee, ${ }^{76}$ C. M. Brown, ${ }^{76}$ D. Fortin, ${ }^{76}$ P. D. Jackson,,${ }^{76}$ R. Kowalewski, ${ }^{76}$ J. M. Roney, ${ }^{76}$ R. J. Sobie, ${ }^{76}$ H. R. Band, ${ }^{77}$ B. Cheng, ${ }^{77}$ S. Dasu, ${ }^{77}$ M. Datta, ${ }^{77}$ A. M. Eichenbaum, ${ }^{77}$ M. Graham, ${ }^{77}$ J. J. Hollar, ${ }^{77}$ J. R. Johnson, ${ }^{77}$ P. E. Kutter, ${ }^{77}$ H. Li, ${ }^{77}$ R. Liu,${ }^{77}$ A. Mihalyi, ${ }^{77}$ A. K. Mohapatra, ${ }^{77}$ Y. Pan,${ }^{77}$ R. Prepost, ${ }^{77}$ P. Tan, ${ }^{77}$ J. H. von Wimmersperg-Toeller, ${ }^{77}$ J. Wu, ${ }^{77}$ S. L. Wu, ${ }^{77}$ Z. Yu, ${ }^{77}$ M. G. Greene, ${ }^{78}$ and H. Neal ${ }^{78}$

\title{
(The BABAR Collaboration)
}

\author{
${ }^{1}$ Laboratoire de Physique des Particules, F-74941 Annecy-le-Vieux, France \\ ${ }^{2}$ Università di Bari, Dipartimento di Fisica and INFN, I-70126 Bari, Italy \\ ${ }^{3}$ Institute of High Energy Physics, Beijing 100039, China \\ ${ }^{4}$ University of Bergen, Inst. of Physics, N-5007 Bergen, Norway \\ ${ }^{5}$ Lawrence Berkeley National Laboratory and University of California, Berkeley, CA 94720, USA \\ ${ }^{6}$ University of Birmingham, Birmingham, B15 2TT, United Kingdom \\ ${ }^{7}$ Ruhr Universität Bochum, Institut für Experimentalphysik 1, D-44780 Bochum, Germany \\ ${ }^{8}$ University of Bristol, Bristol BS8 1TL, United Kingdom \\ ${ }^{9}$ University of British Columbia, Vancouver, BC, Canada V6T $1 Z 1$ \\ ${ }^{10}$ Brunel University, Uxbridge, Middlesex UB8 3PH, United Kingdom \\ ${ }^{11}$ Budker Institute of Nuclear Physics, Novosibirsk 630090, Russia \\ ${ }^{12}$ University of California at Irvine, Irvine, CA 92697, USA \\ ${ }^{13}$ University of California at Los Angeles, Los Angeles, CA 90024, USA \\ ${ }^{14}$ University of California at Riverside, Riverside, CA 92521, USA
}




\author{
${ }^{15}$ University of California at San Diego, La Jolla, CA 92093, USA \\ ${ }^{16}$ University of California at Santa Barbara, Santa Barbara, CA 93106, USA \\ ${ }^{17}$ University of California at Santa Cruz, Institute for Particle Physics, Santa Cruz, CA 95064, USA \\ ${ }^{18}$ California Institute of Technology, Pasadena, CA 91125, USA \\ ${ }^{19}$ University of Cincinnati, Cincinnati, OH 45221, USA \\ ${ }^{20}$ University of Colorado, Boulder, CO 80309, USA \\ ${ }^{21}$ Colorado State University, Fort Collins, CO 80523, USA \\ ${ }^{22}$ Technische Universität Dresden, Institut für Kern- und Teilchenphysik, D-01062 Dresden, Germany \\ ${ }^{23}$ Ecole Polytechnique, LLR, F-91128 Palaiseau, France \\ ${ }^{24}$ University of Edinburgh, Edinburgh EH9 3JZ, United Kingdom \\ ${ }^{25}$ Università di Ferrara, Dipartimento di Fisica and INFN, I-44100 Ferrara, Italy \\ ${ }^{26}$ Florida AEM University, Tallahassee, FL 32307, USA \\ ${ }^{27}$ Laboratori Nazionali di Frascati dell'INFN, I-00044 Frascati, Italy \\ ${ }^{28}$ Università di Genova, Dipartimento di Fisica and INFN, I-16146 Genova, Italy \\ ${ }^{29}$ Harvard University, Cambridge, MA 02138, USA \\ ${ }^{30}$ Universität Heidelberg, Physikalisches Institut, Philosophenweg 12, D-69120 Heidelberg, Germany \\ ${ }^{31}$ Imperial College London, London, SW7 2AZ, United Kingdom \\ ${ }^{32}$ University of Iowa, Iowa City, IA 52242, USA \\ ${ }^{33}$ Iowa State University, Ames, IA 50011-3160, USA \\ ${ }^{34}$ Università di Perugia, Dipartimento di Fisica and INFN, I-06100 Perugia, Italy \\ ${ }^{35}$ Laboratoire de l'Accélérateur Linéaire, F-91898 Orsay, France \\ ${ }^{36}$ Lawrence Livermore National Laboratory, Livermore, CA 94550, USA \\ ${ }^{37}$ University of Liverpool, Liverpool L69 72E, United Kingdom \\ ${ }^{38}$ Queen Mary, University of London, E1 4NS, United Kingdom \\ ${ }^{39}$ University of London, Royal Holloway and Bedford New College, Egham, Surrey TW20 0EX, United Kingdom \\ ${ }^{40}$ University of Louisville, Louisville, KY 40292, USA \\ ${ }^{41}$ University of Manchester, Manchester M13 9PL, United Kingdom \\ $4^{42}$ University of Maryland, College Park, MD 20742, USA \\ ${ }^{43}$ University of Massachusetts, Amherst, MA 01003, USA \\ ${ }^{44}$ Massachusetts Institute of Technology, Laboratory for Nuclear Science, Cambridge, MA 02139, USA \\ ${ }^{45}$ McGill University, Montréal, QC, Canada H3A $2 T 8$ \\ ${ }^{46}$ Università di Milano, Dipartimento di Fisica and INFN, I-20133 Milano, Italy \\ ${ }^{47}$ University of Mississippi, University, MS 38677, USA \\ ${ }^{48}$ Université de Montréal, Laboratoire René J. A. Lévesque, Montréal, QC, Canada H3C 3J7 \\ ${ }^{49}$ Mount Holyoke College, South Hadley, MA 01075, USA \\ ${ }^{50}$ Università di Napoli Federico II, Dipartimento di Scienze Fisiche and INFN, I-80126, Napoli, Italy \\ ${ }^{51}$ NIKHEF, National Institute for Nuclear Physics and High Energy Physics, NL-1009 DB Amsterdam, The Netherlands \\ ${ }^{52}$ University of Notre Dame, Notre Dame, IN 46556, USA \\ ${ }^{53}$ Ohio State University, Columbus, OH 43210, USA \\ ${ }^{54}$ University of Oregon, Eugene, OR 97403, USA \\ ${ }^{55}$ Università di Padova, Dipartimento di Fisica and INFN, I-35131 Padova, Italy \\ ${ }^{56}$ Universités Paris VI et VII, Laboratoire de Physique Nucléaire et de Hautes Energies, F-75252 Paris, France \\ ${ }^{57}$ Università di Pavia, Dipartimento di Elettronica and INFN, I-27100 Pavia, Italy \\ ${ }^{58}$ University of Pennsylvania, Philadelphia, PA 19104, USA \\ ${ }^{59}$ Università di Pisa, Dipartimento di Fisica, Scuola Normale Superiore and INFN, I-56127 Pisa, Italy \\ ${ }^{60}$ Prairie View A\&M University, Prairie View, TX 77446, USA \\ ${ }^{61}$ Princeton University, Princeton, NJ 08544, USA \\ ${ }^{62}$ Università di Roma La Sapienza, Dipartimento di Fisica and INFN, I-00185 Roma, Italy \\ ${ }^{63}$ Universität Rostock, D-18051 Rostock, Germany \\ ${ }^{64}$ Rutherford Appleton Laboratory, Chilton, Didcot, Oxon, OX11 0QX, United Kingdom \\ ${ }^{65}$ DSM/Dapnia, CEA/Saclay, F-91191 Gif-sur-Yvette, France \\ ${ }^{66}$ University of South Carolina, Columbia, SC 29208, USA \\ ${ }^{67}$ Stanford Linear Accelerator Center, Stanford, CA 94309, USA \\ ${ }^{68}$ Stanford University, Stanford, CA 94305-4060, USA \\ ${ }^{69}$ State University of New York, Albany, NY 12222, USA \\ ${ }^{70}$ University of Tennessee, Knoxville, TN 37996, USA \\ ${ }^{71}$ University of Texas at Austin, Austin, TX 78712, USA \\ ${ }^{72}$ University of Texas at Dallas, Richardson, TX 75083, USA \\ ${ }^{73}$ Università di Torino, Dipartimento di Fisica Sperimentale and INFN, I-10125 Torino, Italy \\ ${ }^{74}$ Università di Trieste, Dipartimento di Fisica and INFN, I-34127 Trieste, Italy \\ ${ }^{75}$ Vanderbilt University, Nashville, TN 37235, USA \\ ${ }^{76}$ University of Victoria, Victoria, BC, Canada V8W $3 P 6$ \\ ${ }^{77}$ University of Wisconsin, Madison, WI 53706, USA \\ ${ }^{78}$ Yale University, New Haven, CT 06511, USA
}


We report on a search for the flavor-changing neutral current decays $D^{0} \rightarrow e^{+} e^{-}$and $D^{0} \rightarrow \mu^{+} \mu^{-}$, and the lepton-flavor violating decay $D^{0} \rightarrow e^{ \pm} \mu^{\mp}$. The measurement is based on $122 \mathrm{fb}^{-1}$ of data collected by the BABAR detector at the PEP-II asymmetric $e^{+} e^{-}$collider. No evidence is found for any of the decays. The upper limits on the branching fractions, at the $90 \%$ confidence level, are $1.2 \times 10^{-6}$ for $D^{0} \rightarrow e^{+} e^{-}, 1.3 \times 10^{-6}$ for $D^{0} \rightarrow \mu^{+} \mu^{-}$, and $8.1 \times 10^{-7}$ for $D^{0} \rightarrow e^{ \pm} \mu^{\mp}$.

PACS numbers: 13.20.Fc,14.40.Lb,12.60.-i

In the Standard Model (SM), the flavor-changing neutral current (FCNC) decays $D^{0} \rightarrow e^{+} e^{-}$and $D^{0} \rightarrow \mu^{+} \mu^{-}$[1] are highly suppressed by the GlashowIliopoulos-Maiani (GIM) mechanism [2]. Their decay branching fractions have been estimated to be less than $10^{-13}$ even with long-distance processes included. This prediction is orders of magnitude beyond the reach of current experiments. Furthermore, the lepton-flavor violating (LFV) decay $D^{0} \rightarrow e^{ \pm} \mu^{\mp}$ is strictly forbidden in the SM [3].

Some extensions to the Standard Model can enhance the FCNC processes by many orders of magnitude. For example, $R$-parity violating supersymmetry can increase the branching fractions of $D^{0} \rightarrow e^{+} e^{-}$and $D^{0} \rightarrow \mu^{+} \mu^{-}$ to as high as $10^{-10}$ and $10^{-6}$, respectively [4]. The same model also predicts the $D^{0} \rightarrow e^{ \pm} \mu^{\mp}$ branching fraction to be of the order of $10^{-6}$. The upper bounds on the predicted branching fractions of $D^{0} \rightarrow \mu^{+} \mu^{-}$and $D^{0} \rightarrow$ $e^{ \pm} \mu^{\mp}$ are close to the current experimental sensitivities. As a result, searching for the FCNC and LFV decays in the charm sector is a potential way to test the SM and explore new physics. Similar arguments hold for rare $K$ and $B$ decays, but the charm decay is unique since it is sensitive to new physics coupling to the up-quark sector.

In this paper, we present a search for the decays of $D^{0} \rightarrow e^{+} e^{-}, D^{0} \rightarrow \mu^{+} \mu^{-}$, and $D^{0} \rightarrow e^{ \pm} \mu^{\mp}$. The analysis is based on $122 \mathrm{fb}^{-1}$ of data collected on or near the $\Upsilon(4 S)$ resonance by the BABAR detector at the PEP-II asymmetric $e^{+} e^{-}$collider.

The BABAR detector, which is fully described in [5], provides charged-particle tracking through a combination of a five-layer double-sided silicon micro-strip detector (SVT) and a 40-layer central drift chamber (DCH), both operating in a $1.5 \mathrm{~T}$ magnetic field in order to provide momentum measurements. The identification of charged kaons and pions is achieved through measurements of particle energy-loss $(d E / d x)$ in the tracking system and Cherenkov cone angle $\left(\theta_{c}\right)$ in a detector of internally reflected Cherenkov light. Electrons are identified primarily in a segmented $\mathrm{CsI}(\mathrm{Tl})$ electromagnetic calorimeter, while muons are identified by their penetration through the iron plates of the magnet flux return.

The charmed mesons considered for this analysis originate from the fragmentation of charm quarks in the continuum $e^{+} e^{-} \rightarrow c \bar{c}$ process. There is no advantage in including $D^{0}$ decays from the $B$ mesons because of their higher combinatoric background. The $D^{0} \rightarrow \ell^{+} \ell^{-}(\ell=$ $e, \mu)$ branching ratio is determined by

$$
\mathcal{B}\left(D^{0} \rightarrow \ell^{+} \ell^{-}\right)=S \cdot\left(N_{\mathrm{obs}}-N_{\mathrm{bg}}\right),
$$

where $N_{\text {obs }}$ is the number of $D^{0} \rightarrow \ell^{+} \ell^{-}$candidates observed, $N_{\text {bg }}$ is the expected background and $S$ is the sensitivity factor, defined as:

$$
S \equiv \mathcal{B}\left(D^{0} \rightarrow \pi^{+} \pi^{-}\right) \times \frac{1}{N_{\pi \pi}} \times \frac{\epsilon_{\pi \pi}}{\epsilon_{\ell \ell}} .
$$

Here $\mathcal{B}\left(D^{0} \rightarrow \pi^{+} \pi^{-}\right)=(1.43 \pm 0.07) \times 10^{-3}$ is the $D^{0} \rightarrow \pi^{+} \pi^{-}$branching fraction [6], $N_{\pi \pi}$ is the number of reconstructed $D^{0} \rightarrow \pi^{+} \pi^{-}$decays, $\epsilon_{\ell \ell}$ and $\epsilon_{\pi \pi}$ are the efficiency for the corresponding decay mode. We choose $D^{0} \rightarrow \pi^{+} \pi^{-}$as the normalization mode because it is kinematically similar to $D^{0} \rightarrow \ell^{+} \ell^{-}$and therefore many common systematic uncertainties cancel in the calculation of the efficiency ratio $\epsilon_{\pi \pi} / \epsilon_{\ell \ell}$. The key to the analysis is to reduce backgrounds as much as possible while maintaining a high signal efficiency.

We first outline the general event selection requirements common to all the data samples used in the analysis and later describe tighter optimized criteria specific to each decay modes. A pair of oppositely charged tracks is selected to form a $D^{0}$ candidate. They are fit to a common vertex and only the candidates with fit probability larger than $1 \%$ are retained. Since charmed mesons from $e^{+} e^{-} \rightarrow c \bar{c}$ events are produced with momenta higher on average than those from $e^{+} e^{-} \rightarrow b \bar{b}$ events, a minimum value of $2.4 \mathrm{GeV} / c$ is imposed on the center-of-mass momentum of each $D^{0}$ candidate. In order to further reduce the background, the $D^{0}$ candidate is required to be from a $D^{*+} \rightarrow D^{0} \pi^{+}$decay. The $D^{0}$ candidate and the pion from the $D^{*+}$ are fit to a common vertex with a beam spot constraint. The probability for this fit is required to exceed $1 \%$. The resolution of the mass difference between the reconstructed $D^{*+}$ and $D^{0}$ candidates is approximately $0.25 \mathrm{MeV} / c^{2}$. We require that $|\delta m| \leq 2.0 \mathrm{MeV} / c^{2}$, where $\delta m=m\left(D^{0} \pi^{+}\right)-m\left(D^{0}\right)-145.4 \mathrm{MeV} / c^{2}$. In addition, all the tracks are required to have a minimum number of measurement points in the SVT and the DCH.

We require the electron and muon candidates to have momenta larger than $0.5 \mathrm{GeV} / c$ and $1.0 \mathrm{GeV} / c$ in the laboratory frame respectively. In this range, the average electron and muon efficiencies are about $95 \%$ and $60 \%$, and their hadron misidentification probabilities are measured from $\tau$ decay control samples to be around $0.2 \%$ and $2.0 \%$. Pion identification is also applied to 
the daughters of $D^{0} \rightarrow \pi^{+} \pi^{-}$decays. The corresponding single pion identification efficiency is around $90 \%$. No particle identification (PID) is applied on the soft pion from the $D^{*+}$ decay.

Except for particle identification, the selection criteria applied to the $D^{0} \rightarrow \pi^{+} \pi^{-}$mode are the same as those used for the $D^{0} \rightarrow \ell^{+} \ell^{-}$modes. The signal efficiencies of $D^{0} \rightarrow \ell^{+} \ell^{-}$and $D^{0} \rightarrow \pi^{+} \pi^{-}$are evaluated using a Monte Carlo (MC) simulation. We use PYTHIA [7] for the fragmentation of the produced $c \bar{c}$. The final state radiative effects are simulated for all decays using PHOTOS [8]. The detector response is simulated with GEANT4 [9], and the simulated events are then reconstructed in the same manner as the data.

Due to large final-state radiation and bremsstrahlung backgrounds, the invariant mass distributions, $m_{\ell \ell}$, of $D^{0} \rightarrow e^{+} e^{-}$and $D^{0} \rightarrow e^{ \pm} \mu^{\mp}$ have a low mass tail. We define an asymmetric signal mass window $(1.8045 \leq$ $m_{\ell \ell} \leq 1.8845 \mathrm{GeV} / c^{2}$ ) for all three decay modes. The lower boundary of the signal mass window is chosen to include the majority of the radiative tail of $D^{0} \rightarrow e^{+} e^{-}$ and $D^{0} \rightarrow e^{ \pm} \mu^{\mp}$. The higher boundary corresponds to a little more than $2 \sigma$ of the $D^{0}$ mass resolution measured from the $D^{0} \rightarrow \pi^{+} \pi^{-}$control sample. In order to avoid any possibility of bias, a blind analysis technique has been adopted. All events inside the $D^{0}$ mass window were hidden from inspection until the final event selection criteria were established and all systematic uncertainties were determined.

The $D^{0} \rightarrow \ell^{+} \ell^{-}$background can be taken as a sum of two components: a peaking background from $D^{0} \rightarrow$ $h^{+} h^{-}(h=K, \pi)$ decays and a combinatoric background from other sources. The copious two body hadronic $D^{0} \rightarrow h^{+} h^{-}$decays will mimic the dilepton signals if both hadrons are misidentified as leptons. MC studies show that only the decay $D^{0} \rightarrow \pi^{+} \pi^{-}$contributes in the signal window. The $D^{0} \rightarrow K^{-} \pi^{+}$and $D^{0} \rightarrow K^{+} K^{-}$ backgrounds peak in the lower mass region because of the high kaon mass. To estimate the number of peaking background events, $N_{\mathrm{bg}}^{h h}$, we apply the selection criteria for $D^{0} \rightarrow \ell^{+} \ell^{-}$to MC simulated $D^{0} \rightarrow \pi^{+} \pi^{-}$events with lepton misidentification rates measured from a control sample.

MC studies show that the combinatoric background in both the signal mass window and high mass sideband region $\left(1.9045 \leq m_{\ell \ell} \leq 2.0545 \mathrm{GeV} / c^{2}\right)$ is dominated by the combination of two random leptons. The invariant mass distribution of the random lepton pair is flat. This is indeed consistent with what is observed in the high mass sideband of the data. As a result, the expected combinatoric background in the signal window is just the number of dilepton events in the high mass sideband scaled by the ratio of the width of the signal region to the high mass sideband region.

To further reduce the background, we added a selection on the proper decay time, ct, of the $D^{0}$ candidate, and tightened our selections on the signal mass window and $\delta m$. We determine the optimal selection criteria by maximizing the value $\epsilon_{\ell \ell} / N_{\text {sens }}$, where $N_{\text {sens }}$ is the averaged $90 \%$ confidence level upper limit on the number of observed signal events that would be obtained by an ensemble of experiments with the expected background and no real signal [10]. Studies show that the correlations among the optimized discriminating variables are negligible.

The expected combinatoric background $N_{\mathrm{bg}}^{\text {comb }}$ therefore can be factorized as

$$
N_{\mathrm{bg}}^{\mathrm{comb}}=N_{\mathrm{SB}} \cdot R_{\mathrm{mass}} \cdot R_{\delta m} \cdot R_{c t},
$$

where $N_{\mathrm{SB}}$ is the number of high mass sideband events passing the loose event selection criteria; $R_{\text {mass }}$ is the expected background rejection factor for a given signal mass window; $R_{\delta m}$ and $R_{c t}$ are the expected background rejection factors for the tighter $\delta m$ requirement and the ct requirement respectively. In order to avoid possible bias due to the statistical fluctuation in the high mass sideband, we determine the discriminating variable distribution shape of the combinatoric background from the $\mathrm{MC}$ and $D^{0} \rightarrow \pi^{+} \pi^{-}$control sample. The information is subsequently used to predict the background changes as a function of a particular set of selection criteria rather than by directly examining the data in the high mass sideband. The optimized final selection criteria are summarized in Table I. The estimated numbers of background events are listed in Table II. The proper time

\begin{tabular}{cccc}
\hline \hline Mode & $m_{\ell \ell}\left[\mathrm{GeV} / c^{2}\right]$ & $\delta m\left[\mathrm{MeV} / c^{2}\right]$ & $c t$ \\
\hline$e e$ & $1.8245 \leq m_{e e} \leq 1.8845$ & $|\delta m| \leq 0.6$ & $c t \geq 0$ \\
$\mu \mu$ & $1.8445 \leq m_{\mu \mu} \leq 1.8845$ & $|\delta m| \leq 0.6$ & - \\
$e \mu$ & $1.8445 \leq m_{e \mu} \leq 1.8845$ & $|\delta m| \leq 0.5$ & - \\
\hline \hline
\end{tabular}

TABLE I: The summary of the optimized event selection criteria of $D^{0} \rightarrow \ell^{+} \ell^{-}$.

requirement is found to be useful only for the ee mode. The background in the ee mode is dominated by combinatorials with zero average lifetime and is halved, with a reduction of less than $20 \%$ in signal efficiency, by requiring that the proper time of the $D^{0}$ candidate be positive. Such a requirement is not applied to the $\mu \mu$ mode, where its reflection background has large contribution and a similar lifetime behavior to the $D^{0}$ meson, or to the $e \mu$ mode, where the background is very small.

As an important check of the background estimate, we have compared the expected distribution in the low mass sideband $\left(1.6545 \leq m_{\ell \ell} \leq 1.8045 \mathrm{GeV} / c^{2}\right)$ with the data. The peaking background in the low mass sideband is evaluated from the $D^{0} \rightarrow h^{+} h^{-}$MC sample using precise measurement of the lepton misidentification probabilities. The random lepton pairs are inferred from the events in the high mass sideband. Unlike the upper side, 
the combinatoric background in the low mass sideband has contributions from the combination of two hadrons and the combination of one real lepton with one hadron, where the hadrons are misidentified as leptons. We estimate those backgrounds using MC data and known lepton misidentification rates. We find that the predicted background distributions and levels in the low mass sideband (before and after the optimization of our event selection criteria) have excellent agreement with our observation in the data for all three decay modes.

The number of $D^{0} \rightarrow \pi^{+} \pi^{-}$candidates in the data, $N_{\pi \pi}$, is extracted by fitting their invariant mass distribution with a binned maximum likelihood fit. The signal distribution is modeled as a double Gaussian, and the background distribution is approximated as a linear function. The number of reconstructed $D^{0}$ mesons is found to be between 7000 and 12000 , depending on the selection criteria. The relative uncertainties in $N_{\pi \pi}$ are about $1 \%$.

The invariant mass distribution of the dilepton candidates after applying the optimized event selection criteria is shown in Fig. 1. The number of events observed $\left(N_{\text {obs }}\right)$ and the expected background $\left(N_{\mathrm{bg}}\right)$ are shown in Table II, with no significant excess found in any decay mode.

The largest systematic uncertainty in the signal efficiency ratio $\epsilon_{\pi \pi} / \epsilon_{\ell \ell}$ calculation is due to the PID efficiency. It ranges from $1.2 \%$ for the ee mode to $4.2 \%$ for the $\mu \mu$ mode relative to their efficiency ratio. Other sources of systematic uncertainty are found to be small, including the track reconstruction efficiencies, track momentum resolution and MC statistics.

The systematic uncertainties of the background estimate arise predominantly from the finite data available in the high mass sideband for the $e e$ and $e \mu$ modes. For the $\mu \mu$ mode, a large fraction of the background is produced by misidentified $D^{0} \rightarrow \pi^{+} \pi^{-}$decays. The relative uncertainty associated with the estimate of muon misidentification is found to be about $4.7 \%$.

\begin{tabular}{lccc}
\hline \hline & $D^{0} \rightarrow e^{+} e^{-}$ & $D^{0} \rightarrow \mu^{+} \mu^{-}$ & $D^{0} \rightarrow e^{ \pm} \mu^{\mp}$ \\
\hline$N_{\mathrm{bg}}^{\text {hh }}$ & 0.02 & $3.34 \pm 0.31$ & 0.21 \\
$N_{\mathrm{bg}}^{\text {comb }}$ & $2.21 \pm 0.38$ & $1.28 \pm 0.32$ & $1.93 \pm 0.36$ \\
$N_{\mathrm{bg}}$ & $2.23 \pm 0.38$ & $4.63 \pm 0.45$ & $2.14 \pm 0.36$ \\
$S \quad\left[10^{-7}\right]$ & $2.25 \pm 0.12$ & $4.53 \pm 0.30$ & $3.27 \pm 0.20$ \\
\hline$N_{\text {obs }}$ & 3 & 1 & 0 \\
UL obtained & $1.2 \times 10^{-6}$ & $1.3 \times 10^{-6}$ & $8.1 \times 10^{-7}$ \\
\hline \hline
\end{tabular}

TABLE II: The summary of the number of expected background events $\left(N_{\mathrm{bg}}\right)$, the sensitivity factor $(S)$, number of observed events $\left(N_{\text {obs }}\right)$, and the branching fraction upper limits at the $90 \%$ confidence level for each decay modes. The uncertainties quoted here are total uncertainties. The uncertainty of $N_{\mathrm{bg}}^{h h}$ is negligible for the $e e$ and $e \mu$ decay modes.

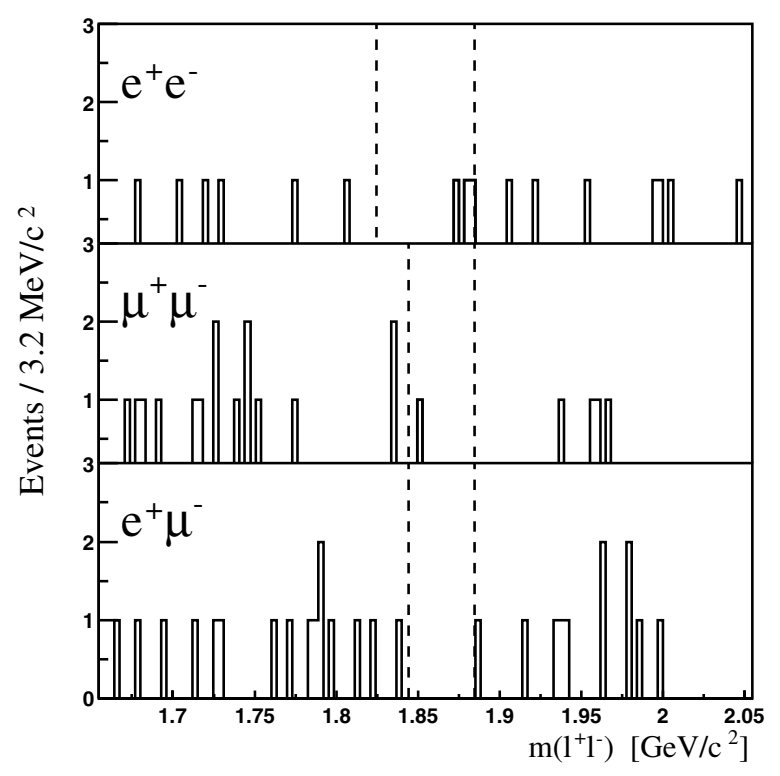

FIG. 1: The dilepton invariant mass distribution for each decay mode. The dashed lines indicate the optimized signal mass window.

The branching fraction upper limits (UL) have been calculated including all uncertainties using an extended version of the Feldman-Cousins method [11]. All of the uncertainties have a negligible effect on the limits. The results are listed in Table II.

In summary, we have performed a search for the FCNC decays $D^{0} \rightarrow e^{+} e^{-}, D^{0} \rightarrow \mu^{+} \mu^{-}$, and the LFV decays $D^{0} \rightarrow e^{ \pm} \mu^{\mp}$ using the BABAR detector. No evidence is found for these decays. The upper limits on the branching fractions at the $90 \%$ confidence level are $1.2 \times 10^{-6}$ for $D^{0} \rightarrow e^{+} e^{-}, 1.3 \times 10^{-6}$ for $D^{0} \rightarrow \mu^{+} \mu^{-}$, and $8.1 \times 10^{-7}$ for $D^{0} \rightarrow e^{ \pm} \mu^{\mp}$. Our result improves the present best limits by a factor of 5 for the ee mode [12], a little less than 2 for the $\mu \mu$ mode [13], and 10 for the $e \mu$ mode [12]. The upper limits for the branching fractions of the $e \mu$ and $\mu \mu$ modes begin to confine the allowed parameter space of R-parity violating supersymmetric models [4].

We are grateful for the excellent luminosity and machine conditions provided by our PEP-II colleagues, and for the substantial dedicated effort from the computing organizations that support BABAR. The collaborating institutions wish to thank SLAC for its support and kind hospitality. This work is supported by DOE and NSF (USA), NSERC (Canada), IHEP (China), CEA and CNRS-IN2P3 (France), BMBF and DFG (Germany), INFN (Italy), FOM (The Netherlands), NFR (Norway), MIST (Russia), and PPARC (United Kingdom). Individuals have received support from CONACyT (Mexico), A. P. Sloan Foundation, Research Corporation, and Alexander von Humboldt Foundation. 
* Now at Department of Physics, University of Warwick, Coventry, United Kingdom

$\dagger$ Also with Università della Basilicata, Potenza, Italy

¥ Also with IFIC, Instituto de Física Corpuscular, CSICUniversidad de Valencia, Valencia, Spain

$\S$ Deceased

[1] Throughout this paper charge conjugation is implied.

[2] S. L. Glashow, J. Iliopoulos and L. Maiani, Phys. Rev. D 2, 1285 (1970).

[3] This process is allowed within extensions to the SM that have non-zero neutrino mass.

[4] G. Burdman, E. Golowich, J. Hewett and S. Pakvasa, Phys. Rev. D 66, 014009 (2002).

[5] B. Aubert et al. [BABAR Collaboration], Nucl. Instrum. Meth. A 479, 1 (2002).
[6] K. Hagiwara et al. [Particle Data Group Collaboration], Phys. Rev. D 66, 010001 (2002).

[7] T. Sjostrand, P. Eden, C. Friberg, L. Lonnblad, G. Miu, S. Mrenna and E. Norrbin, Comput. Phys. Commun. 135, 238 (2001)

[8] E. Barberio and Z. Was, Comput. Phys. Commun. 79, 291 (1994).

[9] S. Agostinelli et al. [GEANT4 Collaboration], Nucl. Instrum. Meth. A 506, 250 (2003).

[10] G. J. Feldman and R. D. Cousins, Phys. Rev. D 57, 3873 (1998).

[11] J. Conrad, O. Botner, A. Hallgren and C. Perez de los Heros, Phys. Rev. D 67, 012002 (2003).

[12] E. M. Aitala et al. [E791 Collaboration], Phys. Lett. B 462, 401 (1999).

[13] I. Abt et al. [HERA-B Collaboration], arXiv:hepex/0405059. 